The degree of association of water was then calculated and the results are shown on the accompanying graph. According to our calculations, near the melting point water is completely associated (that is, every molecule takes part in associated complexes), while at the boiling point only 42 per cent of the molecules behave in this manner. It is interesting to compare the values of $\alpha$ with the fraction of the dipoles, $f$, which have free rotatory power as given by Bernal and Fowler. The values of $(1-f)$, the fraction of the dipoles having no free rotatory power, are given graphically.

At present work is in progress to apply these principles to other substances and to the mechanism of viscosity.

The University,
Edgbaston,

Birmingham, 15. May 28.

${ }^{2}$ Grunberg, L., and Nissan, A. H., Nature, 154, 146 (1944).

2 Keesom, W. H., Phys. Z., 22, 120, 643 (1921); 23, 225 (1922).

'Debye, P., Phys. z., 21, 178 (1920); 22, 302 (1921).

- Bernal, J. D., and Fowler, R. H., J. Chem. Phys., 1, 513 (1933).

\section{The Fitzgerald Contraction as the Origin of our Experience of Time and its Lapse Rate}

LET it be assumed that the origin of the sensation of time and the flux of time lies in the Fitzgerald contraction of brain material. If the brain material is carried relatively to 'space' or 'ether' with constant speed, the sensation will be steady in amount, and so the flow of time will seem to be uniform. For all speeds of motion the contraction is a well-known function of the speed, and it is the most simple postulate that the sensation of the lapse-rate of time is altered correspondingly.

Sir James Jeans has advanced the postulate that the brain is responsive to the one-way increase of entropy. This is possible and perhaps plausible in virtue of the magnitude of the individual brain mechanism attached to a centre of brain response; but it must be remembered that entropy fiux can only be presumed to be steady as an average. There is no such limitation in the case of the Fitzgerald contraction. Its action is one-way unavoidably.

Using Maxwell's equations in the form

$$
\begin{aligned}
& \text { Curl } E+\dot{B}=0 ; \operatorname{div} B=0 ; \\
& \text { Curl } H-\dot{D}=0 ; \operatorname{div} D^{\circ}=0 ;
\end{aligned}
$$

which characterize Schrödinger's recent treatment ${ }^{1}$, that treatment makes clear the possibility of employing Maxwell's electromagnetic theory in the wave mechanics of the present day.

By accepting only data susceptible of ideal or actual demonstration, a possible large and fruitful line of investigation is closed, and we are faced with the complicated picturization of reality as given in terms of relativity and quantum wave mechanics. The existence of ether has been deniod and 'space' enthroned instead. As Sir J. J. Thomson, in forming an ideal working model of the proton in ether, asks: Why not call it ether instead of space and get rid of the difficulty ? Schrödinger's suggestions repeat the demand with the additional simplification of the explicitly stated quality of Maxwellian.
In this connexion we have to regard afresh the striking announcement given to the International Congress of Physics at Paris in 1900 by Kelvin, at once of his renunciation of the longitudinal component of the electric displacement in wave motion through the ether; of his adoption instead of Maxwell's electromagnetic ether; and of his final adherence to Boscovich's theory of the action of forces, of electric attraction or repulsion as providing a basis for the connexion of matter with ether.

In this way, Kelvin brought to a triumphant conclusion in 1900 his work on wave-theory, matter, and ether, begun in his Baltimore Lectures in 1884, and continued intermittently throughout the inter. vening years. This was perhaps the greatest investigation made in theoretical physics since Newton wrote. It vindicated the claim that investigators in physics should not be trammelled by the modern arbitrary restriction. The picture of Nature they give may actually lie nearer its heart.

Now, with this claim made prominent, what, more fully, of the picture of Nature presented by Kelvin ? Primarily, the Maxwellian ether, having negative compressibility and made stable by atomic support in a material medium built up of these atoms. If a physicist of Kelvin's rank was satisfied with that, it should surely give confidence.

Finally, let this ether be that part of the ether contemplated by Schrödinger ${ }^{1}$, corresponding to Maxwell's electromagnetic equations. We need only consider the four dimensions of ether, three mutually perpendicular to each other in our own universe, and one normal to those in the fourth dimension. Regard the ether flux as entering the threedimensional model, contemplated by Schrödinger, from the fourth dimension, and we can regard the working of the model as due to the energy carried into the model by the etherial current. There is no need to contemplate as existing in reality any higher order of ether dimensions than four. To consider space-time only complicates the picture of reality offered to us.

The Weisha, Ninewells,

W. Pendie.

Dundee. May 18.

' Nature, 153, 572 (1944).

\section{Bread in Jersey during German Occupation}

Through Mr. E. F. Mitchell, president-elect of the National Association of Master Bakers, Caterers and Confectioners, we received a sample of bread made in Jersey during the German occupation. The analytical data we obtained on this sample will probably be of interest to many.

The bread was naturally stale and dry, so that it is possible, in view of the moisture content $(10.9$ per cent), to compare the figures obtained directly with those of flour or meal. The bread had the disadvantage of being very dark and unappetizing, while it did not have the advantage of a high vitamin $B_{1}$ content usually associated with dark and longextraction bread. Actually, the vitamin $B_{1}$ figure is no higher than that of the present fairly good coloured 80 per cent flour, which has about 0.80 I.U./gm. The riboflavin is also not especially high; but the nicotinic acid content is substantially higher than that of present-day flour in Great Britain. 